\title{
ANÁLISE DO ÍNDICE DE PERCEPÇÃO DE ESFORÇO NA AVALIAÇÃO DAS PRESSÕES RESPIRATÓRIAS MÁXIMAS EM CRIANÇAS E ADOLESCENTES
}

\author{
ANALYSIS OF THE RATE OF PERCEIVED EXERTION IN THE \\ ASSESSMENT OF MAXIMAL RESPIRATORY PRESSURES IN CHILDREN \\ AND ADOLESCENTS
}

\author{
Juliana Souza de Oliveira ${ }^{1}$, Tania Fernandes Campos ${ }^{2}$, Raíssa de Oliveira Borja ${ }^{1}$, Raphaella \\ Oliveira Elias da Silva ${ }^{1}$, Diana Amélia de Freitas ${ }^{1}$, Laíse Chaves de Oliveira $^{3}$, \\ Karla Morganna Pereira Pinto de Mendonça²
}

\begin{abstract}
Resumo
Objetivo: Analisar o índice de percepção de esforço na avaliação das pressões respiratórias máximas de crianças e adolescentes. Método: Foram avaliadas 144 crianças e adolescentes saudáveis, de sete a onze anos, sendo 63 meninos e 81 meninas, estudantes das redes pública e privada. 0 instrumento utilizado foi o manovacuômetro digital MVD300 (Globalmed ${ }^{\circledR}$, Porto Alegre - RS, Brasil). Foram realizadas no máximo nove manobras, com um minuto de descanso entre cada manobra e cinco minutos entre a medição das pressões inspiratórias e expiratórias máximas. A sensação de esforço percebido foi determinada através da escala de Borg e foi utilizada em quatro momentos: antes das medições das pressões respiratórias máximas, previamente sorteadas; imediatamente após a primeira medição sorteada; após cinco minutos de descanso e imediatamente após a última avaliação. Resultados: O esforço percebido das crianças e adolescentes antes, imediatamente após e cinco minutos após a manovacuometria foi de 7,8 $\pm 1,8 ; 9,6 \pm 2,5$ e $8,5 \pm 2,3$, respectivamente. Houve aumento significativo após a mensuração das pressões inspiratórias e expiratórias máximas $(p<0,001)$. A quantidade de esforço diminuiu significativamente após cinco minutos de descanso da realização das pressões respiratórias máximas $(p<0,05)$. A percepção de esforço foi semelhante para as crianças e adolescentes que realizaram até cinco ou mais manobras. Conclusão: A avaliação das pressões respiratórias máximas, em crianças e adolescentes, mostrou-se um método seguro quanto à percepção de esforço. Sugere-se que a análise do índice de esforço percebido possa ser prática frequente durante a avaliação da força muscular respiratória.
\end{abstract}

Palavras-chave: criança; adolescente; força muscular; músculos respiratórios; teste de esforço; percepção.

\begin{abstract}
Objective: To analyze the rate of perceived exertion in assessing maximal respiratory pressures in children and adolescents. Methods: We evaluated 144 healthy children and adolescents from seven to eleven years old, 63 boys and 81 girls, students from public and private schools. The instrument used was a digital manovacuometer MVD300 (Globalmed ${ }^{\circledR}$, Porto Alegre-RS, Brazil). A maximum of nine maneuvers were carried out, with one minute rest between each maneuver and five minutes between the measurement of maximal inspiratory and expiratory pressures. The rate of perceived effort was determined by Borg scale and was used at four different moments: before measuring maximal inspiratory and expiratory pressures, previously drawn; immediately after the first measurement; after five minutes of rest and immediately after the last assessment. Results: The children and adolescents' perceived exertion before, immediately after and five minutes after assessment was $7.8 \pm 1.8,9.6 \pm 2.5$ and $8.5 \pm 2.3$, respectively. There was a significant increase after measuring maximal inspiratory and expiratory pressures $(p<$ $0.001)$. The amount of effort decreased significantly with five minutes of rest after maximal respiratory pressures $(p<0.05)$. The perceived exertion was similar for children and adolescents who underwent up to five or more maneuvers. Conclusion: The assessment of maximal respiratory pressures in children and adolescents proved to be a safe method regarding perceived exertion. It is suggested that the analysis of the rate of perceived exertion may be a common practice during the evaluation of respiratory muscle strength.
\end{abstract}

Key words: child; adolescent; muscle strength; respiratory muscles; exercise test; perception.

1 Programa de Pós-Graduação em Fisioterapia, Universidade Federal do Rio Grande do Norte - UFRN. Avenida Senador Salgado Filho, 3000, Departamento de Fisioterapia, Campus Universitário, Lagoa Nova, Natal, RN, Brasil.

2 Professora Adjunta, Departamento de Fisioterapia - UFRN. Avenida Senador Salgado Filho, 3000, Departamento de Fisioterapia, Campus Universitário, Lagoa Nova, Natal, RN, Brasil.

3 Fisioterapeuta graduada pela Universidade Federal do Rio Grande do Norte. Avenida Senador Salgado Filho, 3000, Departamento de Fisioterapia, Campus Universitário, Lagoa Nova, Natal, RN, Brasil.

Corresponding author: kmorganna@ufrnet.br

Suggested citation: Oliveira JS et all. Analysis of the rate of perceived exertion in the assessment of maximal respiratory pressures in children and adolescents. J. Hum. Growth Dev. 2012; 22(3): 314-320

Manuscript submitted Mar 25 2012, accepted for publication Aug 302012. 


\section{INTRODUÇÃO}

A avaliação da força muscular respiratória é um importante parâmetro na prática clínica, uma vez que os músculos respiratórios são responsáveis pelo adequado funcionamento do sistema respiratório ${ }^{1,2}$. A avaliação das pressões respiratórias máximas é um método amplamente utilizado para mensurar a força muscular respiratória ${ }^{3}$, a qual pode ser realizada diretamente por meio da manovacuometria ${ }^{4-6}$.

A medição da pressão respiratória máxima é um método rápido, simples, de baixo-custo e nãoinvasivo ${ }^{6-8}$ para obter um índice de força muscular respiratória e envolve especificamente a mensuração da pressão inspiratória máxima $\left(\mathrm{PI}_{\text {Máx }}\right)$ e da pressão expiratória máxima $\left(\mathrm{PE}_{\text {Máx }}\right)^{6,9,10}$. Ao longo dos anos as pressões respiratórias máximas têm sido realizadas em diferentes populações, sendo inclusive bem aceita e reprodutível na população infanto-juvenil. A realização da manovacuometria é útil no acompanhamento de pacientes de diferentes faixas etárias com distúrbios respiratórios e neuromusculares ${ }^{6,7}$, uma vez que tais doenças podem repercutir tanto na força muscular respiratória como na qualidade de vida desta população ${ }^{11}$. Frequentemente em programas de reabilitação o treinamento individual dos músculos respiratórios é baseado na mensuração das pressões respiratórias máximas ${ }^{10}$.

A $\mathrm{PI}_{\text {Máx }}$ e $P E_{\text {Máx }}$ são testes voluntários e esforços-dependentes, ou seja, a mensuração das mesmas depende do esforço do indivíduo avaliado. Portanto, o índice de força muscular pode ser confiável se o esforço for verdadeiramente máximo ${ }^{12,13}$. Desse modo, há a necessidade de uma boa compreensão das manobras a serem realizadas e da vontade do indivíduo em cooperar para a realização de esforços respiratórios realmente máximos ${ }^{13,14}$.

Por se tratar de um teste de esforço, a análise das respostas subjetivas em relação à percepção do esforço, torna-se necessária. O esforço percebido é um conceito definido como a avaliação subjetiva que indica a opinião do sujeito sobre a intensidade do trabalho realizado e tem sido um assunto de atenção crescente na literatura desde $1950^{15,16}$. Os índices de esforço percebido (RPE) podem ser medidos por vários instrumentos de mensuração, como, por exemplo, pela escala de esforço percebido de Borg (RPE de Borg) ${ }^{17}$.

A escala RPE de Borg é a mais comumente utilizada para testes de esforço percebido, o qual contém 15 pontos, variando de 6 a 20 pontos para mensurar a percepção de esforço durante o exercí$\mathrm{cio}^{18}$. A vantagem dessa escala é que as classificações dadas crescem linearmente com a intensidade do exercício, frequência cardíaca e consumo de oxigênio $\left(\mathrm{VO}_{2}\right)^{16-19}$. A escala RPE de Borg aumenta a segurança durante a realização de testes que dependem do esforço e também em outras intervenções nas quais a realização destes testes é necessária. O esforço percebido tem sido utilizado com este propósito em diferentes populações ${ }^{20-22}$.
Dessa forma, a investigação do esforço percebido durante a avaliação das pressões respiratórias máximas é importante na prática clínica, uma vez que não se sabe qual grau de esforço esse teste impõe ao indivíduo. Por ser realizado em crianças e adolescentes com doenças respiratórias crônicas ou neuromusculares, é relevante quantificar esse esforço, a fim de aumentar a segurança da realização do teste. Desse modo, o objetivo é analisar a percepção subjetiva de esforço na avaliação das pressões respiratórias máximas de crianças e adolescentes entre sete e onze anos.

\section{MÉTODO}

\section{Caracterização da pesquisa e aspectos éticos}

Trata-se de de estudo descritivo transversal. Os procedimentos realizados foram aprovados pelo Comitê de Ética em Pesquisa da Universidade Federal do Rio Grande do Norte (parecer 278/2009), de acordo com a resolução 196/96 do Conselho Nacional de Saúde. Os dados foram coletados após a assinatura dos Termos de Consentimento Livre e Esclarecido (TCLE) pelos pais e/ou responsáveis. A população foi composta por crianças e adolescentes de ambos os sexos, na faixa etária de 07 a 11 anos $^{23}$, matriculadas em escolas das redes pública e privada.

\section{Critérios de inclusão}

As crianças e adolescentes elegíveis para participar do estudo não poderiam apresentar: diagnóstico de doença pulmonar crônica, cardiovascular ou neuromuscular ${ }^{24}$; história de traumatismo recente de vias aéreas superiores, tórax ou abdo$\mathrm{me}^{14}$; febre (três semanas) ${ }^{6,14}$, gripe e/ou resfriado na semana anterior ao procedimento ${ }^{5,14}$; história de tabagismo5,6,14; evidente deformidade torácica5,14; problema agudo do ouvido médio ${ }^{14}$, hérnia abdominal ${ }^{14}$; glaucoma ou deslocamento de retina ${ }^{14}$; comprometimento neurológico e/ou cognitivo ${ }^{14,24}$; relato de uso de medicações como glicocorticóides inalatórios ou sistêmicos, mineralocorticóides, estimulantes do sistema nervoso central, barbitúrios ou relaxantes musculares $^{24}$; percentil menor que 5 ou maior ou igual a 85 baseado na curva do IMC em relação à idade e ao sexo de crianças/adolescentes entre 2 e 20 anos de idade proposta pelo National Center for Health Statistics (NCHS), no ano $2000^{25}$.

\section{Critérios de exclusão}

Foram adotados os seguintes critérios de exclusão: não conseguir realizar os procedimentos necessários; desistir de participar da pesquisa; apresentar alguma doença aguda do trato respiratório durante o período de coleta; ou faltar à aula durante todo o período de avaliação na sua escola.

\section{Seleção dos sujeitos}

Os dados foram coletados em 27 escolas selecionadas aleatoriamente por meio de sorteio. Após 
a seleção aleatória das escolas participantes, foram sorteados 50 alunos, em cada escola, sendo 10 de cada idade (de 7 a 11 anos) e, dentre estes, cinco do sexo masculino e cinco do sexo feminino, em cada grupo.

Foi enviado aos pais dos alunos sorteados: 1) uma carta de apresentação do estudo, explicando o que eram os documentos recebidos e como deveriam proceder; 2) o TCLE para os pais, contendo as explicações sobre os objetivos, a importância e os procedimentos do estudo; 3) um questionário que deveria ser respondido pelo responsável, os quais continham perguntas sobre o estado de saúde geral do(a) aluno(a). Após o recebimento da documentação enviada aos pais, foram identificadas as crianças e adolescentes elegíveis para o estudo. Aqueles que estavam aptos a participarem do estudo foram questionados sobre a sua vontade em participar.

\section{Avaliação das Pressões Respiratórias Máximas}

Para aferir as pressões respiratórias máximas foi utilizado o manovacuômetro digital MVD300 (Globalmed ${ }^{\circledR}$, Porto Alegre - RS, Brasil), calibrado de $-300 \mathrm{a}+300 \mathrm{cmH}_{2} \mathrm{O}$, com precisão de $1 \mathrm{cmH}_{2} \mathrm{O}$, conectado a um computador que fornecia ao participante um feedback visual e auditivo. O manovacuômetro digital era acoplado a um filtro biológico descartável e de uso individual.

Inicialmente, após a concordância da criança ou do adolescente apto a participar do estudo, foi sorteada qual a pressão respiratória seria realizada primeiro, ou seja, $\mathrm{PI}_{\text {Máx }}$ ou $\mathrm{PE}_{\text {Máx }}$. Após o sorteio, eram dadas as instruções quanto à realização das pressões respiratórias máximas e, então, iniciado o teste.

As manobras foram demonstradas e explicadas verbalmente para que houvesse o máximo de compreensão possível. Por ser um teste esforço-dependente, foi solicitado ao participante que realizasse o maior esforço que conseguisse e, associado a isso, o avaliador forneceu encorajamento verbal durante a mensuração.

As medições das $\mathrm{PI}_{\text {Máx }}$ ou $\mathrm{PE}_{\text {Máx }}$ foram realizadas de acordo com o método proposto por Souza ${ }^{14}$, por dois avaliadores previamente treinados. Para medição da $\mathrm{PI}_{\text {Máx }}$ foi solicitado que os participantes respirassem normalmente (volume corrente) durante três ciclos respiratórios e após o comando do avaliador, eles deveriam realizar uma expiração máxima (até aproximadamente o volume residual - VR). O voluntário indicou o final da expiração por um gesto previamente combinado. Nesse momento, o avaliador ocluiu o orifício que conecta o sistema com o ar ambiente e solicitou que fosse feita uma inspiração máxima (até aproximadamente a capacidade pulmonar total - CPT).

Para a $\mathrm{PE}_{\text {Máx }}$ as orientações foram semelhantes, diferindo no fato que o indivíduo realizou primeiro uma inspiração máxima e depois uma expiração máxima e que durante esta medição foi realizado, pelo avaliador, uma sustentação manual nas bochechas dos participantes para assegurar uma menor perda de pressão devido à complacência da cavidade oral ${ }^{26}$.

Foram realizadas no máximo 9 manobras para $\mathrm{PI}_{\text {Máx }}$ ou $\mathrm{PE}_{\text {Máx }}$ como sugerido por Domènech-Clar et al. ${ }^{7}$ Destas, foram obtidas pelo menos três manobras aceitáveis (sem vazamento e com duração de pelo menos 2 segundos) e entre as aceitáveis era necessário ter pelo menos duas manobras reprodutíveis (com valores que não diferissem entre si por mais de $10 \%$ do valor mais elevado). No entanto, a última medida não pôde ser a maior, realizando-se outra mensuração caso isso ocorres$\mathrm{se}^{14}$.

Foi dado um minuto de descanso entre a realização de cada manobra e 5 minutos entre a medição das pressões inspiratória e expiratória máximas $^{27}$. Durante todo o teste o voluntário ficou sentado confortavelmente, formando um ângulo de $90^{\circ}$ de quadril e com as costas apoiadas na cadeira. É importante ressaltar que as avaliações ocorreram até o horário de intervalo das escolas, para que fosse evitado que as crianças e os adolescentes realizassem as manobras após terem se alimentado e realizado esforço físico.

\section{Avaliação da percepção de esforço}

O esforço percebido foi determinado através da escala RPE de Borg ${ }^{17}$, que é uma escala vertical quantificada de 6 a 20, onde 6 representa nenhum sintoma e 20 representa o sintoma máximo, proporcionando uma medida individualizada, direta e momentânea da percepção do esforço. A escala possui atributos verbais ao lado dos números para facilitar a compreensão ("sem nenhum esforço"; "extremamente leve"; "muito leve", "leve"; "um pouco intenso"; "intenso"; "muito intenso"; "extremamente intenso"; "máximo esforço") ${ }^{15,17}$. Todas as crianças e os adolescentes receberam orientações quanto à finalidade da escala, como seria executada, e tiveram tempo suficiente para observá-la e se adaptar com as amplitudes dos números.

A escala de Borg foi utilizada em quatro momentos: 1) antes das medições da $\mathrm{PI}_{\text {Máx }}$ ou $\mathrm{PE}_{\text {Máx }}$ inicialmente sorteada pelo participante; 2) imediatamente após a primeira medição sorteada; 3) cinco minutos após o término da primeira avaliação sorteada $\left(\mathrm{PI}_{\text {Máx }}\right.$ ou $\left.\mathrm{PE}_{\text {Máx }}\right) ; 4$ ) imediatamente após o término da última avaliação.

\section{Análise dos dados}

Os dados da amostra foram analisados através do SPSS 17.0 (Statistical Package for the Social Science) atribuindo-se o nível de significância de $5 \%$. A estatística descritiva foi realizada através de médias e desvios padrão. O teste de KolmogorovSmirnov foi utilizado para testar a normalidade dos dados.

Segundo o teste de Kolmogorov-Smirnov, observou-se que apenas os índices de percepção de esforço de $\mathrm{PE}_{\text {Máx }}$ não apresentaram distribuição normal, sendo utilizado o teste não-paramétrico de Wilcoxon para comparar a sensação de esforço ini- 
cial, logo após a realização da $\mathrm{PE}_{\text {Máx }}$ e após cinco minutos de descanso. Para a comparação entres índices de percepção de esforço das crianças e dos adolescentes que realizaram inicialmente a $\mathrm{PI}_{\text {Máx }}$ foi utilizado o teste $\mathrm{t}$ de Student pareado. $\mathrm{O}$ teste $\mathrm{t}$ de
Student não pareado foi utilizado para avaliar a quantidade de esforço necessária entre os participantes que realizaram até cinco manobras com aqueles que realizaram mais de cinco manobras de pressões respiratórias máximas $\left(\mathrm{PI}_{\text {Máx }}\right.$ e $\left.\mathrm{PE}_{\text {Máx }}\right)$.

Tabela 1: Valores do índice de percepção de esforço de Borg antes, imediatamente após a realização das pressões respiratórias máximas e após cinco minutos de repouso $(n=144)$.

$\begin{array}{cccc}\text { RPE de Borg } & \text { Médias e Desvio Padrão } & \text { Mínimo } & \text { Máximo } \\ \text { RPE Inicial } & 7,8 \pm 1,8 & 6 & 13 \\ \text { RPE imediatamente após a PRM } & 9,6 \pm 2,5 & 6 & 17 \\ \text { RPE 5 minutes after PRM } & 8,5 \pm 2,3 & 6 & 19\end{array}$

RPE: índice de percepção de esforço; PRM: pressões respiratórias máximas.

Tabela 2: Comparação entre o índice de esforço percebido (RPE) inicial com os observados imediatamente após as pressões respiratórias máximas ( $n=144)$.

$\begin{array}{lcc} & \text { RPE Inicial } & \text { RPE imediatamente após a MRP } \\ \mathrm{PI}_{\text {Max }} & 7,9 \pm 1,9 & 10,0 \pm 2,8^{* *} \\ \mathrm{PE}_{\text {Max }} & 7,8 \pm 1,7 & 9,3 \pm 2,3^{* *}\end{array}$

RPE: índice de percepção de esforço; PRM: pressões respiratórias máximas; $\mathrm{PI}_{\text {Máx }}$ : pressão inspiratória máxima; $\mathrm{PE}_{\text {Máx }}$ : pressão expiratória máxima;

** significância estatística $(p<0,001)$.

Tabela 3: Comparação entre o índice de esforço percebido imediatamente após as pressões respiratórias máximas com os observados após cinco minutos de repouso $(n=144)$.

\section{RPE immediately after MRP}

$$
\begin{gathered}
10,0 \pm 2,8 \\
9,3 \pm 2,3
\end{gathered}
$$

RPE 5 minutos após o MRP

$$
\begin{gathered}
8,7 \pm 2,4 * * \\
8,5 \pm 2,3 *
\end{gathered}
$$

RPE: Índice de percepção de esforço; PRM: pressões respiratórias máximas; $\mathrm{PI}_{\text {Máx }}$ : pressão inspiratória máxima; $\mathrm{PE}_{\text {Máx }}$ : pressão expiratória máxima;

** significância estatística $(p<0,001)$,

* significância estatística ( $p<0,05)$.

Tabela 4: Comparação entre o índice de esforço percebido (RPE) inicial com os observados depois de cinco minutos de repouso $(n=144)$.

\section{RPE Inicial}

$$
\begin{aligned}
& \mathrm{PI}_{\text {Max }} \\
& \mathrm{PE}_{\text {Max }}
\end{aligned}
$$

$$
\begin{aligned}
& 7,9 \pm 1,9 \\
& 7,8 \pm 1,7
\end{aligned}
$$

RPE 5 minutos após o MRP

$$
\begin{gathered}
8,7 \pm 2,4 \\
8,5 \pm 2,3 *
\end{gathered}
$$

RPE: índice de percepção de esforço; PRM: pressões respiratórias máximas; $\mathrm{PI}_{\text {Máx }}$ : pressão inspiratória máxima; $\mathrm{PE}_{\text {Máx }}$ : pressão expiratória máxima;

* significância estatística $(p<0,05)$.

Tabela 5: Comparação entre as médias do índice de percepção de esforço, de acordo com o número de manobras realizadas, após a avaliação das pressões respiratórias máximas $\left(\mathrm{PI}_{\text {máx }}\right.$ e $\left.P E_{\text {máx }}\right)(n=144)$.

$\begin{array}{lccc} & \text { Número de manobras } & \text { RPE de Borg } & \text { Valor de } \mathbf{p} \\ \mathrm{PI}_{\text {Max }} & \text { Até } 5 \text { manobras } & 10,0 \pm 2,7 & 0,63 \\ & \text { Acima de } 5 \text { manobras } & 10,3 \pm 3,0 & 0,38 \\ \mathrm{PE}_{\text {Max }} & \text { Até } 5 \text { manobras } & 9,6 \pm 2,4 & 9,0 \pm 2,0\end{array}$

$\mathrm{PI}_{\text {Máx }}$ : pressão inspiratória máxima; $\mathrm{PE}_{\text {Máx }}$ : pressão expiratória máxima; RPE: Índice de percepção de esforço. 


\section{RESULTADOS}

Dentre os alunos sorteados e que retornaram a documentação entregue inicialmente, 157 encontravam-se elegíveis para participar do estudo. Destes, 04 foram excluídos por se recusarem a participar, 05 por não compreenderem o comando solicitado, 03 por não conseguirem realizar manobras aceitáveis e reprodutíveis dentro do número máximo de medidas estabelecido no estudo e 01 por ter apresentado febre na semana que seria avaliado. Portanto, a amostra final foi composta por 144 crianças e adolescentes sendo 63 meninos e 81 meninas, com média de idade de $8,8 \pm 1,2$ anos. Os participantes apresentaram, em média, peso de $30,2 \pm 1,2 \mathrm{~kg}$, altura de $1,3 \pm 0,1 \mathrm{~m}$ e IMC de $16,4 \pm$ $1,5 \mathrm{Kg} / \mathrm{m}^{2}$.

Em média, foram necessários aproximadamente cinco esforços máximos para a obtenção de medidas aceitáveis e reprodutíveis para $\mathrm{PI}_{\text {Máx }}$ e $\mathrm{PE}_{\text {Máx }}$ das crianças e adolescentes avaliados. Estas médias foram de 4,6 $\pm 1,7$ e 4,7 $\pm 1,5$ manobras para medir a $\mathrm{PI}_{\text {Máx }}$ e $\mathrm{PE}_{\text {Máx }}$, respectivamente.

A tabela 1 descreve os dados referentes ao esforço percebido dos participantes antes, imediatamente após a realização das manobras das pressões respiratórias máximas e cinco minutos após o repouso. Estes valores estão descritos independentemente de qual manobra foi realizada primeiro.

A tabela 2 compara o índice de esforço percebido inicial com o observado imediatamente após a realização das pressões respiratórias máximas. A tabela 3 compara a percepção de esforço imediatamente após a manovacuometria com o observado após cinco minutos de repouso. A tabela 4, por sua vez, compara o índice de esforço percebido inicial com o observado após cinco minutos de repouso. As comparações estão descritas separadamente para as crianças e os adolescentes que realizaram inicialmente a avaliação da $\mathrm{PI}_{\text {Máx }}$ ou da $\mathrm{PE}_{\text {Máx }}$.

A tabela 5 apresenta a quantidade de esforço percebido pelos participantes que realizaram até cinco manobras comparativamente com aqueles que realizaram mais de cinco manobras para avaliação das pressões respiratórias máximas.

\section{DISCUSSÃO}

A avaliação da força dos músculos respiratórios, por meio da manovacuometria, consiste em um método bem tolerado por crianças e adolescentes, uma vez que, em média, o índice de percepção de esforço subjetivo foi classificado como um esforço "muito leve" 17 na escala RPE de Borg.

Segundo Borg ${ }^{17}$, uma regra adotada por vários clínicos consiste em interromper um teste de esforço em um RPE de 14 ou 15 ("intenso"). Para esse autor, a monitorização do índice de esforço percebido durante um teste de estresse é importante por facilitar a segurança do teste, sendo in- terrompido caso haja alguma intercorrência, principalmente em caso de pacientes e pessoas idosas $^{17}$. Jones e Killian ${ }^{28}$, em estudo posterior, apontaram que no caso de testes de esforço cardiopulmonar, apesar da percepção de esforço do exercício e da percepção de esforço respiratório (medida pela dispneia) aumentarem durante o teste, este deve ser interrompido quando chegar a valores classificados de "intenso" a "muito intenso" na escala modificada de Borg.

Os resultados deste estudo indicam um aumento significativo do índice de percepção de esforço imediatamente após a realização do teste, se comparado com a quantidade de esforço inicial, ou seja, com o participante em repouso antes de realizar qualquer uma das manobras avaliadas. Este incremento pode ser consequente ao fato de a avaliação das pressões respiratórias máximas se tratar de um teste que exige esforços realmente má$x^{x i m o s}{ }^{12,13}$. Coelho et al. ${ }^{29}$ ao compararem a avaliação do esforço percebido de crianças fibrocísticas com crianças saudáveis após a realização de teste de esforço cardiopulmonar, o shuttle walk test, observou que houve um incremento na quantidade de esforço realizada após o teste. No entanto, o cansaço foi maior nas crianças saudáveis. Aquino et al. ${ }^{30}$, ao realizaram o teste de caminhada de seis minutos em uma amostra composta por crianças e adolescentes saudáveis, não observaram diferenças significativas na sensação subjetiva de esforço avaliada pela escala de Borg antes e após a realização deste teste.

Ao analisar o intervalo de repouso entre a realização da $\mathrm{PI}_{\text {Máx }}$ e da $\mathrm{PE}_{\text {Máx }}$ observa-se que este consiste em um intervalo de tempo seguro quanto à percepção subjetiva de esforço. Os resultados apontaram uma redução significativa entre o índice de esforço imediatamente após a realização da manovacuometria e 5 minutos após a mesma. O esforço observado após o descanso pode ser classificado como "extremamente leve"17 na escala de Borg e próximo do índice de esforço inicial, ou seja, antes da avaliação das pressões respiratórias máximas. Volianitis et al. ${ }^{4}$, utilizaram um minuto de descanso entre as manobras de $\mathrm{PI}_{\text {Máx }}$ e três minutos de repouso após a realização da pressão inspiratória máxima, para evitar os efeitos da fadiga. Nascimento et al. ${ }^{27}$ utilizaram um intervalo de cinco minutos entre os esforços inspiratórios e expiratórios máximos ao avaliarem a força muscular respiratória de crianças brasileiras de 7 a 10 anos. Os resultados do presente estudo sugerem que um tempo de pelo menos cinco minutos entre a $\mathrm{PI}_{\text {Máx }}$ e $\mathrm{PE}_{\text {Máx }}$ é suficiente para que o esforço percebido retorne a valores próximos do repouso.

Ao avaliar comparativamente o índice de esforço percebido dos participantes que realizaram até cinco esforços com aqueles que necessitaram de um número superior a cinco manobras para obter uma medida válida e reprodutível para a $\mathrm{PI}_{\text {Máx }}$ e/ou $\mathrm{PE}_{\text {Máx' }}$ observa-se que foram obtidos valores 
semelhantes. Segundo Wen et $\mathrm{al}^{9}{ }^{\text {, }}$ quanto maior o número de manobras realizadas, mais precisa será a avaliação da força dos músculos respiratórios. Domènech-Clar et $\mathrm{al}^{7}$ consideraram adequado o limite de nove manobras para avaliar a força muscular respiratória de crianças e adolescentes. Em contrapartida, Alexandre et al. ${ }^{2}$ preconizaram a realização de 3 a 5 manobras de pressões respiratórias máximas para avaliar a força muscular respiratória. Entretanto, levando em consideração a percepção subjetiva de esforço, a semelhança entre os valores de esforço observados é sugestiva de que o limite de nove manobras é seguro para avaliar a força muscular respiratória de indivíduos a partir dos sete anos de idade.

Algumas limitações foram encontradas no decorrer do estudo e, dentre elas, se destaca as dificuldades quanto ao uso da escala de RPE de Borg com crianças. Algumas crianças, em especial as que se encontravam na faixa etária entre 7 e 8 anos, apresentaram dificuldade na compreensão da escala numérica de 6 a 20, bem como a relação desta com os conceitos verbais associados. Em um estudo recente sobre a acurária da escala de Borg modificada no exercício de crianças e adolescentes entre 6 e 18 anos com fibrose cística, Hommerding ${ }^{18}$

\section{REFERÊNCIAS}

1. Costa D, Gonçalves HA, Lima LP, Ike D, Cancelliero KM, Montebelo MIL. Novos valores de referência para pressões respiratórias máximas na população brasileira. J Bras Pneumol. 2010;36(3):306-12. doi: 10.1590/ S1806-37132010000300007.

2. Alexandre BL, Araújo SG, Machado MGR. Pressões respiratórias máximas. In: Machado, MGR. Bases da fisioterapia respiratória: terapia intensiva e reabilitação. Rio de Janeiro: Guanabara Koogan; 2008. p. 111-24.

3. Simões RP, Deus AP, Auad MA, Dionísio J, Mazzonetto M, Borghi-Silva A. Pressões respiratórias máximas em indivíduos saudáveis sedentários de 20 a 89 anos da região central do Estado de São Paulo. Rev Bras Fisioter. 2010;14(1):60-7. doi: 10.1590/S141335552010000100010.

4. Volianitis S, McConnel AK, Jones DA. Assessment of maximum inspiratory pressure. Respiration. 2001;68(1):22-7. doi: 10.1159/ 000050458.

5. Parreira VF, França DC, Zampa CC, Fonseca MM, Tomich GM, Britto RR. Pressões Respiratórias Máximas: valores encontrados e preditos em indivíduos saudáveis. Rev Bras Fisioter. 2007;11(5):361-8. doi: 10.1590/S141335552007000500006.

6. Neder JA, Andreoni S, Lerarioet MC, Nery LE. Reference values for lung function tests. II. Maximal respiratory pressures and voluntary observou que a percepção subjetiva de dispneia, através da escala modificada de Borg, apresentava maior precisão com crianças em idade superior a 9 anos. Estudos anteriores ${ }^{16,17}$ já haviam considerado a idade como um aspecto capaz de influenciar nas respostas ao índice de esforço percebido.

A inexistência de estudos prévios que tenham analisado o esforço percebido durante a avaliação da força dos músculos respiratórios pode ser considerada outra limitação, uma vez que dificultou a discussão do presente estudo. No entanto, isto serviu como incentivo à realização dessa pesquisa, uma vez que a avaliação da sensação de esforço é um método simples e proporciona maior segurança na realização de testes esforços-dependentes, devendo ser utilizada nas diversas populações, tanto em adolescentes quanto em crianças.

Assim, a avaliação da força muscular respiratória de crianças e adolescentes, por meio das pressões respiratórias máximas, mostra-se um método seguro quanto à percepção de esforço. O intervalo de cinco minutos entre a realização da $\mathrm{PI}_{\text {Máx }}$ e $\mathrm{PE}_{\text {Máx }}$ foi considerado adequado e o limite de nove manobras para a avaliação destas pressões não altera de forma significativa a percepção de esforço da criança e do adolescente.

ventilation. Bras J Med Biol Res. 1999;32(6):719-27. doi: 10.1590/S0100879X1999000600007.

7. Domènech-Clar R, López-Andreu JA, CompteTorrero L, De Diego-Damiá A, Macián-Gisbert $V$, Perpiñá-Tordera $M$, et al. Maximal static respiratory pressures in children and adolescents. Pediatr Pulmonol. 2003;35(2):126-32. doi: 10.1002/ppul.10217.

8. Freitas DA, Borja RO, Ferreira GMH, Nogueira PAMS, Mendonça KMPP. Equações preditivas e valores de normalidade para pressões respiratórias máximas na infância e adolescência. Rev Paul Pediatr. 2011;29(4):656-62. doi: 10.1590/S010305822011000400028

9. Wen AS, Woo MS, Keens TG. How many maneuvers are required to measure maximal inspiratory pressure accurately. Chest. 1997;111(3):802-7.

doi:10.1378/chest.111.3.802.

10. Tomalak W, Pogorzelski A, Prusak J. Normal values for maximal static inspiratory and expiratory pressures in healthy children. Pediatr Pulmonol. 2002;34(1):42-6. doi: 10.1002/ ppul.10130.

11. Trinca MA, Bicudo IMP, Pelicione MCF. A interferência da asma no cotidiano das crianças. Rev Bras Crescimento Desenvolv Hum. 2011;21(1):70-84.

12. American Thoracic Society/European Respiratory Society. ATS/ERS statement on respiratory muscle testing. Am J Respir Crit 
Care Med. 2002;166(4):518-624. doi: 10.1164/rccm.166.4.518.

13. Aldrich TK, Spiro P. Maximal inspiratory pressure: does reproducibility indicate full effort? Thorax. 1995;50(1):40-43. doi: 10.1136/thx.50.1.40.

14. Souza RB. Pressões respiratórias estáticas máximas. J Pneumol. 2002;28 Suppl 3:S155165.

15. Burkhalter N. Evaluación de la escala Borg de esfuerzo percibido aplicada a la rehabilitación cardiaca. Rev Latinoam Enferm. 1996;4(3):6571. doi: 10.1590/S0104-11691996000300006.

16. O'Sulivan SB. Perceived Exertion: A Review. Phys Ther. 1984;64(3):343-6.

17. Borg G. Escalas de Borg para a dor e o esforço percebido. São Paulo: Manole; 2000.

18. Hommerding PX. Acurácia da escala de Borg modificada no exercício de crianças e adolescentes com fibrose cística [dissertação]. Porto Alegre: Universidade Federal do Rio Grande do Sul, Faculdade de Medicina; 2008.

19. Chen MJ. Criterion-related validity of the Borg ratings of perceived exertion scale in healthy individuals: a metaanalysis. J Sports Sci. 2002; 20(11):873-99. doi: $10.1080 / 026404102320761787$

20. Carlsen KH, Engh G, Mørk M. Exercise-induced bronchoconstriction depends on exercise load. Respir Med. 2000;94(8):750-5. doi: 10.1053/ rmed.2000.0809.

21. Macedo TMF, Oliveira KMC, Melo JBC, Medeiros MG, Medeiros Filho WC, Ferreira GMH, et al. Treinamento muscular inspiratório em crianças com leucemia aguda: resultados preliminares. Rev Paul Pediatr. 2010;28(4):352-8. doi: $10.1590 / S 0103-05822010000400011$.

22. Ziegler B, Rovedder PM, Oliveira CL, de Abreu e Silva F, de Tarso Roth Dalcin P. Repeatability of the 6-minute walk test in adolescents and adults with cystic fibrosis. Respir Care. 2010;55(8):1020-5.
23. WHO, World Health Organization. Young People's Health - a Challenge for Society. Report of a WHO Study Group on Young People and Health for All. Technical Report Series 731. Geneva: WHO, 1986.

24. Harik-Khan RI, Wise RA, Fozard JL. Determinants of maximal inspiratory pressure: the Baltimore longitudinal study of aging. Am J Respir Crit Care Med. 1998;158(5 Pt 1):145964.

25. Kuczmarski RJ, Ogden CL, Guo SS, GrummerStrawn LM, Flegal KM, Mei Z, et al. 2000 CDC growth charts for the United States: Methods and development. National Center for Health Statistics. Vital Health Stat. 2002;11(246): 1-190.

26. Oliveira KMC, Macêdo TMF, Borja RO, Nascimento RA, Medeiros Filho WC, Campos TF et al. Força muscular respiratória e mobilidade torácica em crianças e adolescentes com leucemia aguda e escolares saudáveis. Rev Bras Cancerol. 2011;57(4):511-7.

27. Nascimento RA, Campos TF, Melo JBC, Raíssa de Oliveira Borja, Freitas DA, Mendonça KMPP. Obtained and predicted values for maximal respiratory pressures of Brazilian children. ] Hum Growth Dev. 2012;22(1): 166-72. doi: 10.1186/1755-7682-5-4.

28. Jones NL, Killian KJ. Exercise limitation in health and disease. N Engl J Med. 2000;343(9): 632-41.

29. Coelho CC, Aquino ES, Almeida DC, Oliveira GC, Pinto RC, Rezende IMO, et al. Análise comparativa e reprodutibilidade do teste de caminhada com carga progressiva (modificado) em crianças normais e em portadoras de fibrose cística. J Bras Pneumol. 2007;33(2):168-74. doi: $10.1590 / S 1806-37132007000200011$.

30. Aquino ES, Mourão FAG, Souza RKV, Glicério BM, Coelho CC. Análise comparativa do teste de caminhada de seis minutos em crianças e adolescentes saudáveis. Rev Bras Fisioter. 2010;14(1):75-80. doi: 10.1590/S141335552010000100012 . 